\title{
Über die ruhenden und die präsynaptischen Phasen der Reduktionsteilung.
}

\author{
Von \\ Noboru Takamine. \\ (Contributions to Cytology and Genetics from the Morphological \\ Department of Botany, Science College, Imperial \\ University, Tokyo. No. 24.)
}

Viele Forscher meinen, dass die Chromosomen eines Kerns nicht nur während der Mitose, sondern auch oft im Ruhestadium fortbestehen, obwohl ihre Individualität in verschiedenem Grade sichtbar ist. In meiner früheren Mitteilung (16) beschrieb ich, dass Cardiocrinum cordatum in der meiotischen Kernteilung vom Ruhestadium bis zur Synapsis ungepaarte Chromatinklïmpchen aufwies und die Chromatinklïmpchenzahl mit der Chromosomenzahl nicht übereinstimmte.

Nun werde ich über dieses Problem noch einige neuere Ergebnisse meiner Untersuchungen über Materialien aus derselben Familie sowie aus anderen Familien darbieten, mit besonderer Bezugnahme auf die Prochromosomen und ihr Verhältnis zur Chromosomenzahl.

\section{Methode.}

Sämtliche Materialien fixierte ich mit Flemmingscher ChromOsmium-Essigsäure-Lösung in Bonner und in starker Konzentration, ausgenommen, dass ich für Senecio, Lactuca und Anemone meistenteils Bournsche Lösung und C'ARnoy's Alkohol-Chloroform-Essigsäure-Gemisch benutzte. Diese gaben verhältnismässig gute Resultate.

Zur Färbung benutzte ich Heidenhain's Eisen-Alaun-Hämatoxylin, aber auch Safranin-Lichtgrün für Rhodea und Cardio- 
crinum. Diese Färbung erwies sich beim Stadium der Prophase besonders brauchbar, indem sie die Kerne rot, das Plasma und die Zellmembran bläulich darbot. Die mit letzterem Färbungsmittel gefärbten Präparate wiesen einen ziemlich deutlichen Färbungsunterschied $z$ wischen den Chromatinkliumpchen und den wabigen Teilen in den Kernen auf.

Kernkörperchen und Chromatinkliumpchen färben sich gewöhnlich hellrot, ihr wabiger Teil hingegen zeigt sich schwach dunkelgrün oder fast farblos. Aber selten färben sich die Kernkörperchen grünlich, während die Chromatinklümpchen in den präsynaptischen Phasen hellrot erscheinen, wobei ich die Kernkörperchen und die Chromatinklïmpchen deutlich unterscheiden konnte. Leider verblasst die grüne Farbe dieser Präparate nach mehreren Monaten.

\section{Geschichtliches.}

Strasburger (14) behauptete, dass ,,die Chromätinkörner, die wir direkt $z u$ sehen bekommen, noch nicht letzte Erbeinheiten darstellen, so könnten wir sie nur als grössere oder kleinere Pangenosomenkomplexe gelten lassen, und ich möchte vorschlagen, sie als ,Pangenosomen' zu bezeichnen." Er zeigt, dass die dichteren Partien im Gerüstwerke eines ruhenden Kerns von Galtonia und Funkia mit der erwarteten Zahl von Chromosomen nicht übereinstimmt.

Gregolre und Wygaerts behaupten nur indirekt das Fortbestehen der Individualität der Chromosomen im ruhenden Kerne. Gregorre meint, dass das Chromatin nicht nur in Knoten, sondern auch im Netzwerk enthalten ist. Nach LundegardH (4) ist die Anzahl der Chromatinkliumpchen gross und schwankend bei Trollius, und man kann nicht von individualisierten Chromatinelementen im Ruhestadium sprechen, während sie bei Calendula und Achillea paarweise geordnet sind und die Zahl der Chromatinklïmpchen etwa mit der somatischen Chromosomenzahl übereinstimmt. Er schlug an anderer Stelle den Namen ,Caryotin' statt der bisher benutzten Termini, ,Chromatin' und ,Linin' vor. 
Rosenberg (11) erwähnt, dass bei einigen Pflanzen die Prochromosomen in bestimmter Anzahl im ruhenden Kerne vorkommen, nämlich die Zahl derselben mit der Chromosomenzahl dieser Pflanzen übereinstimmt. Bei einigen anderen Pflanzen betont er dagegen, dass die Feststellung der Chromatinkliumpchenzahl sehr schwierig ist. Er unterscheidet am Kerngeruist des Ruhekerns passend zwei Typen, nämlich Capsella- und FritillariaTypus. Er sagt, dass bei Capsella-Typus die Kerne im Ruhezustande scheinbar bestimmte Chromatinkörner besitzen, während bei Fritillaria-Typus die Kerne im Ruhestadium zahlreiche kleine Chromatinkörner aufweisen. Solche Kerne kommen oft unter den Liliaceae und Ranunculaceae vor.

Auch bemerkte er, dass ïberhaupt bei Fritillaria-Typus die Chromosomen in den Mitosen sehr lang gestreckte fadenförmige Segmente, bei Capsella-Typus kurze, rektanguläre oder kugelige Stäbchen darbieten.

Overton (8) bezeichnete zuerst die dichteren Chromatinanhäufungen als Prochromosomen, welche im Ruhestadium von Thalictrum purpurascens sich paarweise $z \mathfrak{u}$ vereinigen scheinen. Er bemerkte auch, dass die Zahl der Prochromosomen dieser Pflanzen gleich der der Chromosomen in somatischen Kernen ist.

AlLen (1) behauptete, dass in dem präsynaptischen Stadium von Lilium canadense die Zahl der Chromatinkliumpchen grösser ist, als die der Chromosomen.

Dieselben Verhältnisse wurden auch von Miyake (6) bei der Untersuchung von Lilium martagon gefunden.

Laibach (3) meint, dass die Prochromosomen, welche er als Zentren der Chromosomen formuliert denkt, nicht ganz die Chromosomen darstellen.

Mottier (7) bemerkte, dass bei Podophyllum peltatum und Lilium martagon die Zahl der Chromatinkliimpchen grösser ist als die der somatischen Chromosomen.

Digby (2) erweist, dass bei Crepis virens die Zahl der Chromatinsubstanzen im prämeiotischen Kerne umbestimmt ist, und in dem präsynaptischen Stadium bemerkt er, „Crepis virens shows two alternative series of presynaptic phases, and these may occur in the same inflorescence. In the one series, the 
chromatic contents are aggregated into definite chromatic bodies, while in the other, the chromatin is more finely distributed as small beads throughout the nuclear reticulum."

Rosen (13) zeigt in seiner Arbeit, dass in den Ruhekernen von Scilla sibirica Eunucleolen und Pseudonucleolen, welche letztere er als die Hauptmasse der Kernfäden ausgemacht denkt, durch die Färbung unterschieden werden können.

\section{Eigene Beobachtungen.}

\section{Ginkgo biloba L.}

Der Kern bietet im Ruhestadium eine sehr reiche chromatische Substanz in mässig dünnen, vom Hämatoxylin fast ungefärbten Gerüstwerken dar. Der Verlauf der Ruhe bis vor der Synapsis ist etwa ähnlich mit dem von Rhodea Arten. Die Chromatinkliimpchen verteilen sich zahlreich in den Kernen und zeigen unpaarweise Anordnung (Fig. 1). Ich kann ein Verhältnis dieser Zahl mit der reduzierten Chromosomenzahl 12 schwer auffinden.

Rhodea japonica Roth. et KunTH. Cardiocrinum cordatum (Thunb.) Makino.

Die Kerne der jungen Pollenmutterzellen von Rhodea zeigen im Ruhestadium eine feine gerïstartige Grundmasse, die von Safranin Lichtgrün schwach dunkelgrün oder fast farblos gefärbt ist. In dieser Grundmasse tritt eine Anzahl grösserer und kleinerer Körnchen auf, die ziemlich starke Affinität für das Safranin zeigen, ebensowie bei Cardiocrinum cordatum. Bei Betrachtung von Fig. 2 fällt auf, dass das Kerngerüst aus Chromatinklïmpchen von verschiedenen Grössen und aus Linin besteht. Alle Chromatinkliumpchen sind anscheinend nur von gleichmässig gefärbten Substanzen gebaut. Sie ordnen sich nicht zu Paaren, wie in Fig. 2, 3, 4, 6, 7, 8, obwohl es bei einigen von ihnen so scheint, als ob sie paarweise gekoppelt wären. Die Gebilde dieser Körner bieten keine Regelmässigkeit dar, und die Anzahl derselben ist nicht leicht zu bestimmen, da sie chromatinreich sind. Wie aus den Figuren 7 u. 8 zu er- 


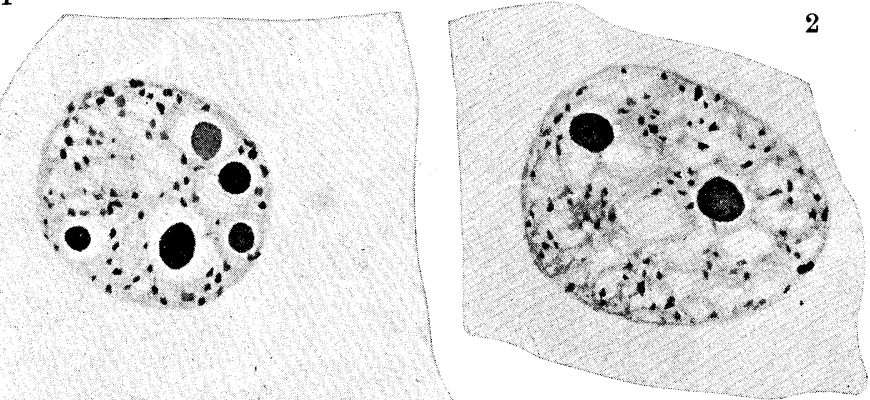

5
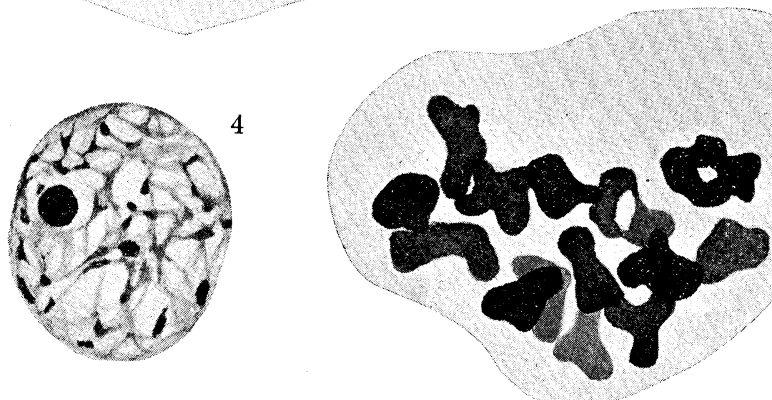

7

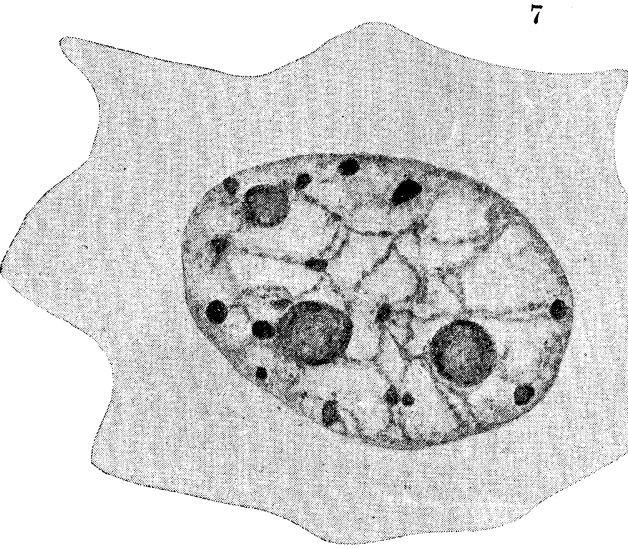

Sämtliche Figuren sind mit Hilfe des ABBE'schen Zeichenapparates gezeichnet, unter Benutzung von ZEISS hom. Imm. $\frac{1}{12}$ und Comp. Ocular 12.

Alle zeigen den Kern der Pollenmutterzellen.

Textfig. 1, Ruhender Kern von Ginkgo biloba. 2-5, Rhodea japonica.

2, Ruhender Kern.

$3-4$, Präsynapsis.

5 , Diakinese.

6-8, Cardiocrinum cordatum

6, Ruhender Kern.

8

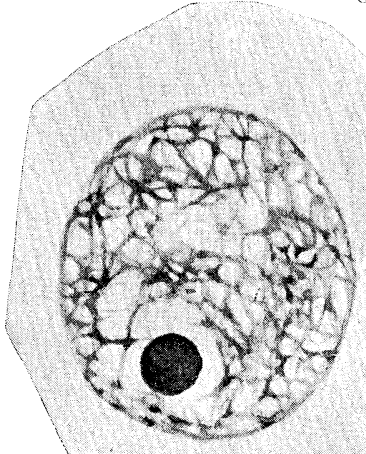

7-8, Präsynapsis.

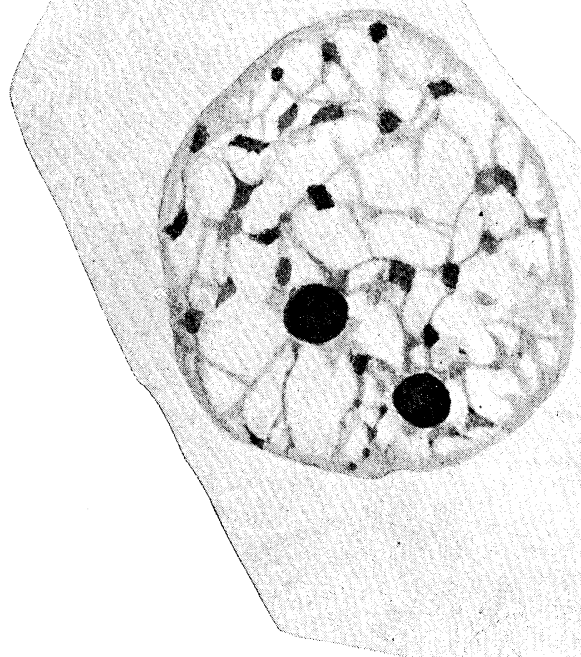


9

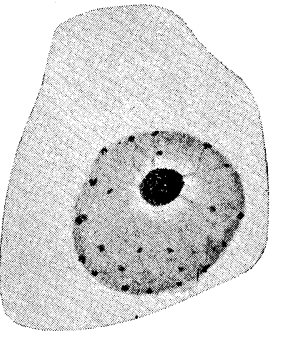

12

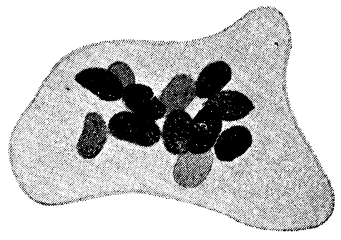

10

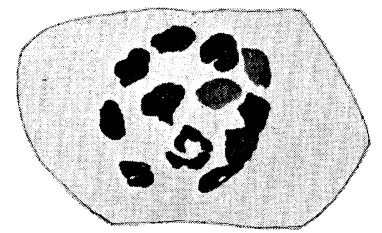

13

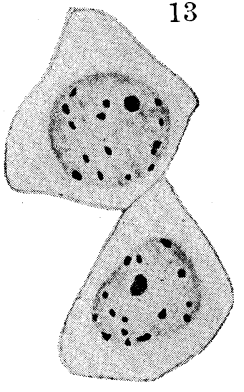

17
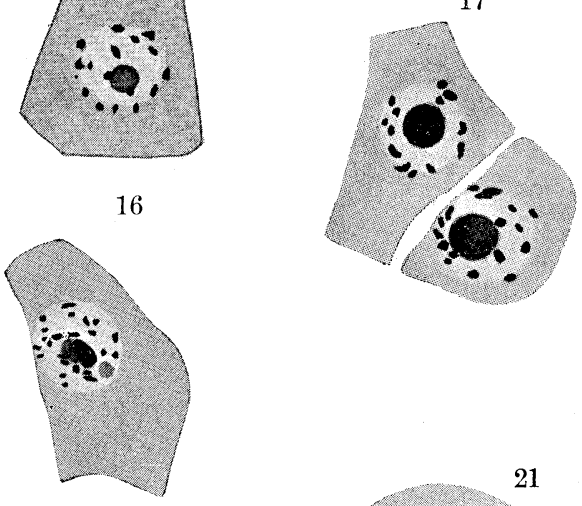

20

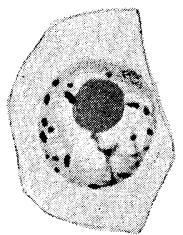

15

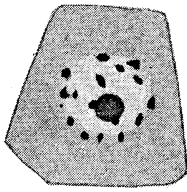

16

21

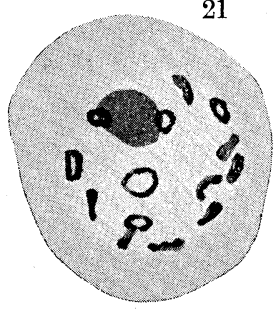

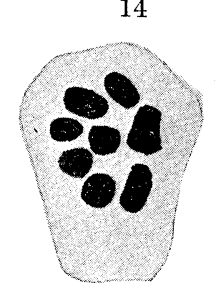

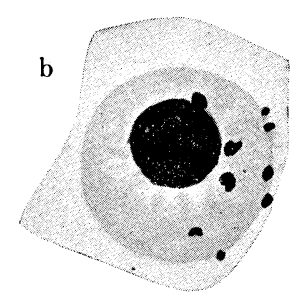

a

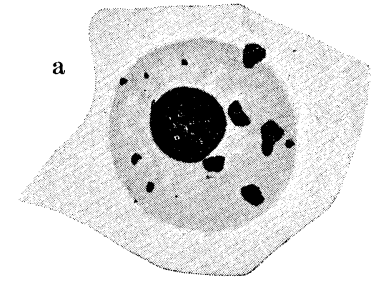

23

22
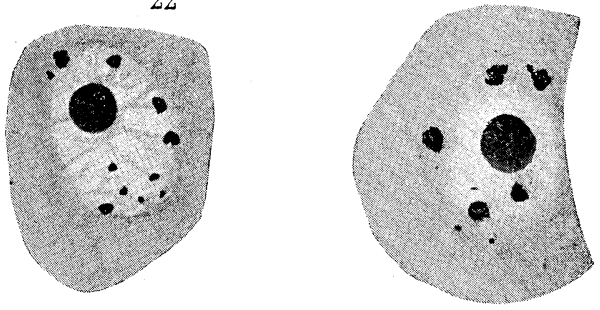

Textfig. 9-10, Spiranthes australis. 11-12, Adonis davurica. 13-14, Anemone japonica.

9, Ruhender Kern. $11 \mathrm{a}, \mathrm{b}$, Ruhende Kerne. 13, Ruhender Kern. 15-16, Ruhende Kerne von Wasabia japonica. 17, Ruhender Kern der Archesporen. 19, Metaphase in Polansicht. 20, Ruhender Kern. 22-23, Ruhende Kerne mit paarigen Prochromosomen von Lactuca lanceolata var. platyphylla.
10, Metaphase in Polansicht. 12, Diakinese.

14, Metaphase in Polansicht. 17-19, Kern von Brassica campestris. 18, Ruhender Kern. 20-21, Senecio vulgaris. 21, Diakinese.

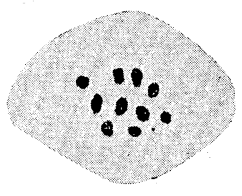

23 
sehen ist, scheinen die Chromatinklümpchen sich im Laufe der Prophase zu vergrössern, und oft sogar zwei oder drei von ihnen sich mit einander $z u$ vereinigen.

Die Kernkörperchen sind zuerst gewöhnlich 3 oder 4 an Zahl, später vereinigen sie sich zu einem oder zwei grossen Kernkörperchen. In Fig. 8 zeigen sich zwei oder drei Punkte in der Peripherie der Chromatinklïmpchen. Ob diese Punkte nur Endfiguren von Lininfäden sind oder nicht, darüber kann ich nichts Bestimmtes sagen. Wenn der Kern weiter wächst, erscheinen die dünnen Lininfäden deutlicher, wobei ich schwach, aber stets bemerken kann, dass mehrere dunkel aussehende Chromatinkörner in einer Reihe in den Lininfäden liegen. Ob diese Chromatinkörner aus den oben erwähnten Chromatinklïmpchen hinaus gebracht, oder ob sie von Anfang an in den Grundmassen der Lininfäden verborgen waren, kann ich nicht ohne weiteres bestimmen. Aber ich möchte sagen, dass es nicht gänzlich unwahrscheinlich ist, dass die dunkel aussehenden Chromatinkörner schon von Anfang an in den Grundmassen der Lininfäden, abgesondert von den Chromatinklïmpchen, verborgen gelegen haben, da ich sie, wenn auch nur schwierig, schon in dem Netzwerkgerüste wahrnehmen konnte, ehe die Chromatinklümpchen sich miteinander vereinigten oder zu dem Netzwerkgerïste zu verschmelzen anfingen, nämlich in einem Stadium, wenn die rundlichen Umrisse der Chromatinklïmpchen sich noch klar beobachten lassen.

Wenn ich bemerke, dass die Zahl der Chromatinklümpchen in der präsynaptischen Phase nicht festgesetzt werden könne, und dass die Chromatinkliimpchen bei der Verschmelzung in dem Netzwerkgeruiste sich in verchiedenen Richtungen ausdehnen, so fiel es mir doch auf, dass die Chromatinkliimpchen den grössten Teil der Substanz der Chromosomen ausmachen, aber doch nicht die ganze Anlage der Chromosomensubstanzen darbieten. Wie in Fig. 5 gezeigt, ist die reduzierte Chromosomenzahl von Rhodea japonica 14.

Ich versuchte auch an Spiranthes australis, ob sie deutliche Prochromosomen darbieten oder nicht, aber ich bemerkte nur undeutlich paarweise Anordnung der Chromatinklïmpchen und 
kein Verhältnis derselben mit ihrer Haploid-Chromosomenzahl 12, wie in Fig. 10 gezeigt.

Adonis davurica Ledeb., Anemone japonica S. et $Z$.

Oft begegnete ich mehr oder weniger grossen Chromatinkliumpchen in dem Ruhestadium von Adonis davurica (Fig. 11a). Obwohl dabei deren Zahl umbestimmt ist, so bieten sich selten einige paarweise geordnete Chromatinkliumpchen dar (Fig. 11b). Diese grossgeformten Klïmpchen treten oft in somatischen Kernen auf. Nicht selten zeigen die Chromatinkliumpchen in denselben Materialien verschiedene Gestalten, die wenigstens teilweise der Natur und dem Eindringungsgrad der Fixierungsflüssigkeit zuzuschreiben sind. Dies bewies LUNDEGÅRDH (7) schon von Allium und Vicia durch verschiedene Fixierungsmittel. Diese Chromatinklümpchenzahl lässt sich schwer feststellen und so auch die Verhältnisse $z$ wischen dieser und der reduzierten Chromosomenzah! 12. Auch bei Anemone japonica stimmt die Chromatinklümpchenzahl mit ihrer Haploid-Chromosomenzahl 8 nicht überein.

Wasabia japonica Matsum. Brassica campestris L.

Einige von mir untersuchte Cruciferen bieten etwas andere Umstände als die schon erwähnten Lilium-Arten dar.

In den Kernen dieser Pflanzen sind die Chromatinklümpchen in den sehr schwach gefärbten, fast farblosen Kerngerüsten deutlich sichtbar (Fig. 17, 18).

In den ruhenden Kernen der Archesporen von Brassica campestris treten oft etwa 16-20 Chromatinkliumpchen deutlich auf, wie in Fig. 17 dargestellt ist, aber es liess sich nicht feststellen, ob diese Zahl mit der somatischen Chromosenzahl übereinstimmte.

In den Pollenmutterzellen zählte ich etwa 10 oder selten gerade 10 Chromatinkliumpchen, was genau mit dieser reduzierten Chromosomenzahl übereinstimmt. Leider konnte ich die reduzierte Chromosomenzahl von Wasabia nicht bestimmen, da ich nur sehr wenig Material zur Verfügung hatte.

Senecio vulgaris L. Lactuca lanceolata var. platyphylla (Franch. et Say.) Makino.

Einige cytologische Forschungen über diese Probleme sind bei den Compositen schon gemacht worden. DisBy bemerkte, 
dass in den prämeiotischen ruhenden Kernen von Crepis virens die Zahl der chromatischen Körner inkonstant ist, und sich zwischen eins und sechs bewegen dïrfte. Auch behauptete er, dass die Chromatinkörner die paarweise Anordnung durch Spaltung herstellen, niemals durch Annäherung. Dagegen beschrieb Rosenberg in den somatischen ruhenden Kernen von Crepis virens 6 Prochromosomen, und auch 6 mehr oder weniger deutlich zu Paaren vereinigte Prochromosomen in den Pollenmutterzellen.

Bei Senecio vulgaris konnte ich diese bestimmte Chromatinkliimpchenzahl nicht finden (Fig. 20), aber bei L. lanceolata var. platyphylla sah ich, wiewohl selten, deutlich 5 (i.e. die reduzierte Chromosomenzahl) Chromatinklïmpchen in den ruhenden und zwar Kernen, bei einigen von ihnen paarweise Anordnung (Fig. 23).

Die Ergebnisse meiner obigen Untersuchungen lassen sich folgendermassen zusammenfassen :

1) Im Ruhestadium der Pollenmutterzellen von Ginkgo biloba, Rhodea japonica und Cardiocrinum cordatum zeigt sich eine feine gerüstartige Grundmasse, in der eine Anzahl grössere und kleinere Körnchen auftreten, die als Chromatinkliumpchen anzusehen sind. Die Zahl dieser Chromatinkliumpchen ist bei obigen Materialien inkonstant. Bei diesen Materialien ist es nicht gänzlich unwahrschcinlich, dass das Chromatin nicht nur in den Chromatinklïmpchen, sondern auch in dem Netzwerkgerüst als kleine Körner vorhanden ist.

2) Im Ruhestadium der Pollenmutterzellen von Adonis davurica und Anemone japonica zeigen sich die Chromatinklïmpchen etwas deutlicher abgesondert von der Lininsubstanz als in den oben erwähnten Fällen, aber ich konnte das Verhältnis zwischen der Zahl der Chromatinklümpchen und der der Chromosomen nicht deutlich genug erkennen, manchmal sind jene viel zahlreicher als die Chromosomen.

3) Einige von mir untersuchte Materialien von Cruciferen zeigen die Prochromosomengestalt deutlich, besonders Brassica campestris.

In seltenen Fällen stimmt die Zahl der Chromatinklümpchen 
mit der reduzierten Chromosomenzahl 10 uberein, wobei ausserdem einige sehr kleine Chromatinkliumpchen bemerkbar sind.

4) In den ruhenden Kernen von L. lanceolata var. platyphylla zeigt sich selten paarweise Anordnung der grössern Chromatinklümpchen und zwar in Uebereinstimmung ihrer Zahl mit der reduzierten Chromosomenzahl 5, nebst einer Anzahl von kleinen Chromatinklümpchen.

5) So entspricht Rosenberg's Fritillaria-'Typus dem meinigen von Ginkgo, Rhodea, Cardiocrinum, Adonis, Anemone, und sein Capsella-Typus annährend dem meinigen von Brassica, Lactuca, aber doch nicht ganz mit ihm übereinstimmend.

Beim Eintreten des Ruhestadiums des Kerns findet bei gewissen Pflanzen eine Zerteilung der individuellen Chromosomen in zahlreiche Kliumpchen und Körner statt, dagegen bei anderen Pflanzen, wie bei L. lanceolata var. platyphylla, nur in eine kleine Anzahl derselben; mit anderen Worten: der Zerteilungsgrad der Chromosomen ist verschieden.

Die vorliegende Arbeit wurde unter Leitung des Herrn Prof. Dr. FujII ausgeführt, dem ich zu herzlichstem Dank verpflichtet bin.

\section{Iiteraturverzeichnis.}

1. Aluen, C. E. Das Verhalten der Kernsubstanzen während der Synapsis in den Pollenmutterzellen vou Lilium Canadense. (Jahrb. f. wiss. Bot. Bd. XLII. 190j).

2. Drgby, L. A critical study of the cytology of Crepis virens. (Arch. f. Zellforsch. Bd. XII. 1904).

3. LabBaCH, F. Zur Frage nach der Individualität der Chromosomen im Pflanzenreich. (Beih. z. Bot. Centralbl. Bd. XXII. 1907).

4. LundegÅRH, H. Ueber Reduktionsteilung in den Pollenmutterzellen einiger dicotylen PHanzen. (Svensk Bot. Tidsk. Bd. III. 1909).

5. Derselbe, Das Caryotin im Ruhekern und sein Verhalten bei der Bildung und Auflösung der Chromosomen. (Arch. f. Zellforsch. Bd. IX. 1913).

6. Mryake, K. Ueber Reduktionsteilung in den Pollenmutterzellen einiger Monokotylen. (Jahrb. f. wiss. Bot. Bd. XLII. 1905).

7. Motriek, D. M. The Development of the heterotypic Chromosomes in Pollenmothercells. (Ann. of Bot. Vol. XXI. 1907).

8. Overton, J. B. Ueber Reduktionsteilung in den Pollenmutterzellen einiger Dikotylen. (Jahrb. f. wiss. Bot. Bd. XLII. 1905).

9. Derselbe, On the organisation of the nuclei in the pollenmothercells of certain plants, with especial reference to the permanence of the chromosomes. (Ann. of Bot. Bd. XXIII. 1909). 
10. Rosenberg, O. Ueber den Bau des Ruhekerns. (Svensk Bot. Tidsk. Bd. III. 1909).

11. Derselbe, Ueber die Individualität der Chromosomen im Pflanzenreich. (Flora Bd. XCIII. 1904).

12. Derselbe, Zur Kenntnis der präsynaptischen Entwicklungsphasen der Reduktionsteilung. (Svensk Bot. Tidsk. Bd. I. 1907).

13. Rosen, F. Beiträge zur Kenntnis der Pflanzenzellen. (Beitrïge z. Piol. d. Pflanzen. Bd. V. 1892).

14. Strasburger, E. Typische und allotypische Kernteilung. (Jahrb. f. wiss. Bot. Bd. XLII. 1905).

15. Stout, A. B. The individuality of the Chromosomes and their serial Arrangement in Carex aquatilis. (Arch. f. Zellforsch. Bd. IX. 1913).

16. N. Takamine. Über die Prophasen der Kernteilungen von Cardiocrinum cordatum (Thunb.) Makrno. Bot. Mag. Bd. XXIX, Heft 337. 1915 (Japanisch). 